Revista de

Contabilidade e Organizações

www.rco.usp.br
DOI: http://dx.doi.org/10.11606/issn.1982-6486.rco.2020.170418
Journal of

Accounting and

Organizations

\title{
Efeitos do debate do sistema de mensuração de desempenho e do conflito cognitivo na inovação ambidestra
}

\section{The effects of the performance measurement system debate and cognitive conflict on ambidextrous innovation}

Januário José Monteiro ${ }^{\mathrm{a}}$, Ilse Maria Beuren ${ }^{\mathrm{a}}$

${ }^{a}$ Universidade Federal de Santa Catarina

Palavras-chave

Sistema de mensuração de desempenho. Conflito cognitivo.

Inovação ambidestra.
Keywords

Performance measurement system.

Cognitive conflict.

Ambidextrous innovation.

\section{Resumo}

$\overline{\text { Este estudo segue o fluxo de pesquisas que investigaram a relação entre o sistema de }}$ mensuração de desempenho e a inovação, mas difere ao considerar a inovação um construto multidimensional. Este estudo analisa os efeitos do debate do sistema de mensuração de desempenho e do conflito cognitivo na inovação ambidestra. Uma pesquisa de levantamento foi realizada com gestores de empresas listadas na Brasil Bolsa Balcão (B3), conforme o ranking dos valores de pesquisa e desenvolvimento e do ativo intangível, e obteve-se 124 respostas válidas. Para testar as hipóteses utilizou-se a técnica de modelagem de equações estruturais. Os resultados revelaram que o debate do sistema de mensuração de desempenho influencia positivamente na inovação ambidestra e que o conflito cognitivo modera essa relação. Conclui-se que as discussões entre os gestores sobre as medidas de desempenho permitem a resolução de problemas, busca pela aprendizagem, criatividade e inovação, principalmente quando intensificado pelo conflito cognitivo.

\begin{abstract}
This study follows the flow of research that investigated the relationship between the performance measurement system and innovation but differs in considering innovation as a multidimensional construct. This study analyzes the effects of the performance measurement system debate and cognitive conflict on ambidextrous innovation. A survey was conducted with managers of companies listed in Brazil Bolsa Balcão (B3), according to the ranking of research and development values and intangible assets, and 124 valid answers were obtained. To test the hypotheses we used the structural equation modeling technique. The results revealed that the performance measurement system debate positively influences ambidextrous innovation and that cognitive conflict moderates this relationship. It is concluded that discussions between managers about performance measures allow the problem-solving, pursuit of learning, creativity, and innovation, especially when intensified by cognitive conflict.
\end{abstract}

\section{Implicações práticas}

A pesquisa auxilia as empresas a fomentarem um ambiente de debate das medidas de desempenho entre os gestores, principalmente nas agendas de elaboração dos planos e ações, pois geram aprendizagem e aumentam a capacidade de criação de novas ideias, que permite a organização se antecipar para liderar a concorrência.

Copyright (C) 2020 FEA-RP/USP. Todos os direitos reservados

Autor correspondente: Tel. (48)3721-3891

E-mail: januariomonteiromonteiro@gmail.com (J. J. Monteiro); ilse.beuren@gmail.com (I. M. Beuren)

Universidade Federal de Santa Catarina, Campus Reitor João David Ferreira Lima, s/n - Trindade, Florianópolis/SC - 88040-900, Brasil. 


\section{INTRODUÇÃO}

Pesquisas que abordam sobre Sistemas de Controle Gerencial (SCG) e inovação têm sido recorrentes, dada a sua importância para a gestão da inovação nas organizações (Bisbe \& Otley, 2004; Bedford, 2015). Estudos nessa linha focalizaram principalmente nos efeitos dos SCG na inovação de produtos (Davila, 2000; Bisbe \& Otley, 2004; Bisbe \& Malagueño, 2009), inovação de maneira geral (Henri, 2006; Davila, Foster \& Oyon, 2009; Chenhall, Kallunki \& Silvola, 2011) e recentemente em inovação ambidestra (Bedford; Bisbe \& Sweeney, 2019). Observam-se diferentes abordagens na literatura (Davila, 2000), de um lado, estudos apontam que os SCG dificultam o processo de Pesquisa e Desenvolvimento (P\&D), de outro lado, que os SCG estimulam a capacidade criativa do indivíduo e de aprendizagem da organização, que levam ao aumento da inovação (Davila et al., 2009; Bisbe \& Malagueño, 2015; Lopez-Valeiras, Gonzalez-Sanchez \& Gomez-Conde, 2016).

Davila et al. (2009) comentam que os SCG fornecem estruturas estáveis que facilitam o processo de comunicação entre os indivíduos na organização e proporcionam liberdade equilibrada, que favorecem a criatividade e a busca pela inovação. Lopez-Valeiras et al. (2016) afirmam que as pesquisas sobre SCG têm limitado seu foco ao uso interativo do SCG na investigação de seu impacto na inovação. Estas escolhas são justificadas pelo fato do uso interativo possibilitar o diálogo permanente entre os gestores, maior criatividade e capacidade de inovar da organização (Simons, 1995; Bisbe \& Malagueño, 2015). Pesquisas pregressas forneceram evidências de que o uso interativo do SCG influencia positivamente na capacidade de inovação das organizações (Henri, 2006; NaranjoGil \& Hartmann, 2007; Bisbe \& Malagueño, 2015; Lopez-Valeiras et al., 2016)

Chenhall (2003) destaca que a missão dos SCG é fornecer informações financeiras e não financeiras com vistas em melhorar o processo decisório. O presente estudo atenta-se ao sistema de mensuração de desempenho (Performance Measurement Systems - PMS), que compreende um conjunto de medidas financeiras e não financeiras usadas para auferir o desempenho (Lamberti \& Noci, 2010). O PMS é considerado nesta pesquisa um antecedente da inovação em razão de ser um importante mecanismo para o alcance da inovação (Davila, Epstein \& Shelton, 2012). Outra motivação para este alvo é o conhecimento limitado sobre o impacto do PMS na capacidade de inovação das organizações (Bedford et al., 2019).

Pesquisas anteriores já consideraram o uso interativo de SCG na análise da capacidade de inovação das empresas (Lopez-Valeiras et al., 2016), um construto multidimensional, que captura o diálogo e debate, incertezas estratégicas e intensidade do uso pelos gestores operacionais (Bedford \& Spekle, 2018; Bedford et al., 2019). Nesta pesquisa, focaliza-se no diálogo e debate, dado que o interesse da proposta está centrado na comunicação entre os gestores, tendo como construto o debate do PMS (Bedford et al., 2019). Esta construção reflexiva consente o debate e livre fluxo da informação entre os gestores e ajuda a promover a inovação (Lopez-Valeiras et al., 2016), pertinente ao propósito desta pesquisa.

Diferente das pesquisas anteriores que consideraram a inovação como um construto unidimensional (Bisbe \& Otley, 2004; Bisbe \& Malagueño, 2009), neste estudo analisa-se a inovação sob duas perspectivas: incremental e radical. A inovação incremental é definida como pequenas mudanças tecnológicas efetuadas nos produtos ou serviços capazes de suprir as necessidades das partes interessadas (Garcia \& Calantone, 2002). A inovação radical é definida como mudanças significativas nos produtos ou serviços, capazes de criar demandas não conhecidas a priori (Jansen, Van Den Bosch \& Volberda, 2006). A busca por mudanças em produtos e ou serviços de forma incremental e radical, simultaneamente, é considerada inovação ambidestra (Sarkees \& Hulland, 2009).

Para que o PMS promova inovação ambidestra é indispensável que as medidas de desempenho sejam apresentadas de maneira equilibrada (Bedford et al., 2019), e propiciem o debate entre os gestores em decorrência das divergências de opiniões quanto ao uso das medidas de desempenho (Bedford et al., 2019). Dreu e West (2001) afirmam que a discordância entre os gestores no processo de tomada de decisão agrega qualidade nas decisões. Para Simons (1995), o debate permanente das medidas de desempenho (controle interativo) tende a estreitar o relacionamento entre os decisores e aumentar a comunicação entre eles, o que leva aos objetivos organizacionais.

Estas divergências de opiniões entre gestores no processo decisório são consideradas conflitos cognitivos e sua ocorrência no ambiente de trabalho é comum e benéfica ao processo decisório (Dreu \& West, 2001; Dreu, 2006). Assim, os indivíduos com objetivos comuns, embora possuam opiniões diferentes, buscam gerenciar os conflitos de modo que as divergências sejam legitimadas e gerem ideias inovadoras (Tjosvold, Poon \& Yu, 2005; Desivilya, Somech \& Lidgoster, 2010). Presume-se que o conflito cognitivo dos gestores intensifique a relação entre o debate do PMS e a inovação ambidestra. 
Bisbe e Otley (2009) alertaram sobre a necessidade de mais pesquisas relativas a diferentes tipos de inovação para aumentar a compreensão sobre a relação entre os SCG e a inovação. Bedford (2015) aponta que não está claro se os diferentes padrões de controle são igualmente eficazes para diferentes tipos de inovação adotados simultaneamente. Bedford et al. (2019) sugerem que se adote o conflito cognitivo como elemento moderador na relação entre o PMS e a inovação. Assim tem-se como questão de pesquisa: Quais os efeitos do debate do PMS e do conflito cognitivo na inovação ambidestra (incremental e radical)?

A relevância do estudo está em compreender como as organizações usam o PMS na busca da inovação incremental e radical. Assim, contribui-se com o corpo da literatura que relaciona SCG e inovação (Davila, 2000; Bisbe \& Otley, 2004; Bisbe \& Malagueño, 2009; Davila et al., 2009), ao seguir o fluxo que conjectura relação positiva entre o PMS e a inovação, e ao adotar o conflito cognitivo como variável que intensifica essa relação. Para Bedford (2015), o conhecimento sobre os SCG empregados na busca pela inovação ambidestra é limitado. Neste sentido, a pesquisa avança na compreensão sobre como as medidas de desempenho são usadas em organizações com múltiplos objetivos de inovação (incremental e radical), sugerindo que o PMS usado de forma interativa, por meio de debate entre os gestores, aumenta a inovação ambidestra.

Agrega-se ainda à literatura ao considerar que o conflito cognitivo está presente nas agendas de discussão entre os gestores e produz efeito positivo, como a geração de ideias inovadoras. Essas evidências reforçam os argumentos em relação aos benefícios do conflito cognitivo apontados na literatura (Dreu, 2006). Para a prática gerencial agrega-se ao buscar evidências empíricas que sustentem os argumentos de que, por meio de debate do PMS, as organizações fomentam um ambiente de maior aproximação entre os gestores, com vistas na resolução de problemas, melhorias tecnológicas e liderança da concorrência.

\section{REFERENCIAL TEÓRICO}

\subsection{Sistema de mensuração de desempenho e inovação ambidestra}

A literatura que trata sobre SCG é vasta e foi conceituada de diferentes maneiras. Anthony (1965) concebeu o SCG como o processo pelo qual os gestores asseguram que os recursos são obtidos e utilizados de modo eficiente com o intuito de alcançar os objetivos organizacionais. Flamholtz, Das e Tsui (1985) definiram SCG como um conjunto de normas e padrões que influenciam o comportamento dos indivíduos na organização. Malmi e Brown (2008) entendem o SCG como um sistema de valores e regras que direcionam o comportamento do indivíduo para o alcance dos objetivos da organização. Para Chenhall (2003), o SCG não apenas está voltado ao fornecimento de informações formais, financeiras e quantificáveis, mas também não-financeiras, por serem úteis ao processo decisório.

As informações financeiras e não-financeiras fornecidas pelos SCG são usadas para aferir o desempenho da organização nos seus diversos níveis e comportam o PMS (Lamberti \& Noci, 2010). Ferreira e Otley (2009) reconhecem a dificuldade de se estabelecer uma definição para o PMS, no entanto, conceituam-no como mecanismos, processos e sistemas formais e informais usados pela organização com a finalidade de transmitir os principais objetivos para auxiliar no processo estratégico e de gerenciamento contínuo. O PMS tem se mostrado um importante mecanismo para o alcance da inovação organizacional (Davila et al., 2012). Neste sentido, Bedford et al. (2019) propõem o debate do PMS, que está relacionado ao conteúdo da informação e à troca da mesma entre os gestores.

Para Bedford et al. (2019), o debate do PMS melhora o processo de tomada de decisão nas empresas em que seus gestores lidam com duplas demandas de inovação. Nesta perspectiva, as empresas utilizam recursos para fornecer seus produtos e serviços de maneira eficiente aos atuais clientes, além de inovar para atender às necessidades futuras dos mesmos e potenciais clientes (Sarkees \& Hulland, 2009). Quando as empresas direcionam seus esforços em projetos de P\&D com essas duplas demandas, visam à inovação ambidestra (Sarkees \& Hulland, 2009; Bedford et al., 2019). Embora pesquisas como de Bedford (2015) tenham examinado o uso do SCG em relação a diferentes tipos de inovação, o fizeram de forma separada. Por exemplo, investigaram a inovação radical ou incremental, de produto ou de processo, administrativa ou tecnológica (Kim, Kumar \& Kumar, 2012).

Para que o PMS possa fomentar a inovação ambidestra é indispensável que seja equilibrado, ou seja, constituído de diversas medidas (Bedford et al., 2019). A inovação incremental se dá pelas pequenas mudanças no design, função, preço, quantidade e características dos produtos ou serviços, para atender as necessidades dos clientes (Garcia \& Calantone, 2002; Sarkees \& Hulland, 2009), enquanto a inovação radical implica adotar novas tecnologias capazes de criar demandas não conhecidas pelos clientes e mercado (Jansen et al., 2006; Sarkees \& Hulland, 2009). 
A inovação incremental apresenta baixo nível de risco e oferece menos benefícios (Koberg, Detienne \& Heppard, 2003), já a inovação radical acarreta alto nível de risco e incerteza. Diante de múltiplas prioridades estratégicas, Dekker, Groot e Schoute, (2012) recomendam um PMS com diversidade de medidas de desempenho.

Um PMS equilibrado, com diversas medidas de desempenho, permite que durante a elaboração dos planos e ações ocorram discussões entre os gestores com vistas na melhoria das decisões (Bedford et al., 2019). O debate das medidas de desempenho corresponde à forma como o PMS é usado para facilitar a troca de informação e aumento da eficácia durante o processo de tomada de decisões pelos gestores (Bedford et al., 2019). Estudos anteriores provaram que o uso interativo do SCG fomenta a inovação, por propiciar o debate permanente e agendas recorrentes, o que envolve estreito relacionamento de trabalho e comunicação frequente (Simons, 1995). O autor argumenta ainda que o debate das medidas de desempenho fornece novas ideias de inovação, e leva à criação de novos produtos ou serviços.

O uso interativo do PMS aumenta a capacidade de inovar da organização (Simons, 1995; Widener, 2007; Bisbe \& Malagueño, 2009; Malagueño \& Bisbe, 2010). Pesquisas apontam relação positiva entre o uso interativo do SCG e a inovação (Henri, 2006; Naranjo-Gil \& Hartmann, 2007; Widener, 2007; Bisbe \& Malagueño, 2015). Henri (2006) fornece evidências que o uso interativo do controle influencia na aprendizagem e na inovação. Naranjo-Gil e Hartmann (2007) apontam que o uso interativo do SCG influencia na inovação de serviços. Widener (2007) aduz que o controle interativo aumenta a aprendizagem organizacional, o que é coerente com a inovação. Para Bisbe e Malagueño (2015), o uso interativo do SCG promove a criatividade, o que influencia na inovação. Lopez-Valeiras et al. (2016) observam que o uso interativo do SCG alavanca a inovação.

Depreende-se que o uso interativo do SCG consente o debate e o livre fluxo das informações, permitindo que os gestores procurem meios alternativos para resolver problemas em seus processos e desenvolver novas propostas que promovam a inovação (Lopez-Valeiras et al., 2016). Ao possibilitar diálogo e debate, o uso interativo do SCG facilita a avaliação das normas e padrões organizacionais e da eficiência dos processos que legitimam as inovações na organização (Bisbe \& Otley, 2004; Lopez-Valeiras et al., 2016). Bedford et al. (2019) fornecem evidências de que o uso interativo do PMS aumenta a inovação ambidestra. A partir da literatura conjectura-se a seguinte hipótese:

$\mathbf{H}_{1}:$ O debate do PMS tem efeito direto e positivo na inovação ambidestra.

\subsection{Efeito moderador do conflito cognitivo na relação entre debate do PMS e inovação ambidestra}

A literatura que aborda sobre o impacto do SCG na inovação é crescente (Simons, 1995; Davila, 2000; Bisbe \& Otley, 2004; Davila et al., 2009; Davila et al., 2012; Bedford, 2015), e revela que o SCG desempenha um importante papel na gestão da inovação (Bisbe \& Otley, 2004). Simons (1995) aponta a capacidade dos SCG se articularem para possibilitar a inovação. Neste aspecto, Davila et al. (2012) alertam que os gestores devem escolher medidas de desempenho adequadas para alavancar a capacidade de inovar nas empresas

Chenhall et al. (2011) consideram que o SCG exerce influência na inovação de maneira indireta. Outras variáveis podem ajudar na melhor compreensão dessa relação (Bisbe \& Otley, 2004; Bedford, 2015). Bedford et al. (2019) sugerem que o conflito cognitivo seja testado como variável moderadora da relação entre o SCG e a inovação. Parece razoável considerar o conflito cognitivo como variável moderadora da relação entre o debate do PMS e a inovação, visto que é inevitável sua ocorrência nas relações entre equipes de trabalho em uma organização (Dreu, 2006).

O conflito cognitivo envolve distribuição de recursos, procedimentos, julgamento e interpretações de fatos (Jehn, 1995). O conflito cognitivo é alvo deste estudo por estar relacionado ao conteúdo das informações, diferenças de ideias, opiniões e pontos de vista (Clercp, Menguc \& Auh, 2009).

Para Jehn (1997), o conflito cognitivo fortalece o relacionamento entre os membros da equipe e permite a conscientização das diferenças de opiniões. Tais ocorrências aumentam a compreensão mútua e a comunicação, o que permite que visões divergentes sobre os procedimentos de trabalho sejam legitimadas e promovam a inovação (Tjosvold et al., 2005; Desivilya et al., 2010).

Dreu (2006) aponta que o conflito cognitivo pode ser benéfico para a aprendizagem e o P\&D de novos produtos. West (2002) observou que no processo de tomada de decisão o debate entre os gestores proporciona ideias mais inovadores, haja vista que o conflito durante o debate das decisões promove criatividade individual e inovação. 
Bedford et al. (2019) argumentam que o conflito cognitivo aumenta a probabilidade dos gestores de topo analisarem de maneira crítica e reflexiva as ideias e opiniões. Reforça-se, assim, a necessidade do debate entre os indivíduos e a legitimação dessas discussões na resolução de problemas (Tjosvold et al., 2005), com vistas na eficácia das decisões dos gestores e da organização como um todo (Amason \& Mooney, 1999).

Em organizações que buscam múltiplos objetivos de inovação (incremental e radical) é importante que haja indivíduos com diferentes opiniões (Jehn, 1995; Simons \& Peterson, 2000; Dreu, 2006), cujas divergências exigem diálogo e debate entre os gestores (Calton \& Payne, 2003). Assim, espera-se que o debate das medidas de desempenho leve ao aumento da inovação ambidestra e seja intensificado pelo conflito cognitivo. Simons (1995) afirma que o diálogo permanente, concebido como uso interativo, impacta na capacidade de inovação da organização. Bedford et al. (2019) alertam que o debate do PMS provoca tensões entre os indivíduos, o que pode ser intensificado pelo conflito cognitivo. Tais tensões permitem que os gestores aprendam e criem oportunidades para apresentar ideias inovadoras e potencializem a inovação na organização (West, 2002). Desse modo, conjectura-se a seguinte hipótese.

$\mathbf{H}_{2}$ : $\mathrm{O}$ conflito cognitivo modera a relação positiva entre o debate do PMS e a inovação ambidestra (incremental e radical).

Face à base teórico-empírica e hipóteses formuladas, destaca-se o modelo teórico da pesquisa na Figura 1.

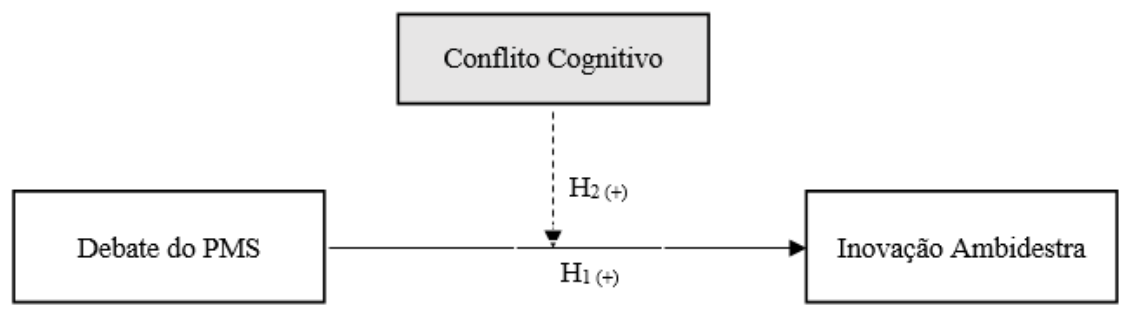

Figura 1. Modelo teórico da pesquisa

Fonte: elaboração própria.

Em linhas gerais, conjectura-se que o debate do PMS tem relação positiva com a inovação ambidestra e que o conflito cognitivo intensifica essa relação.

\section{METODOLOGIA DA PESQUISA}

\subsection{População e Amostra}

O levantamento dos dados foi realizado com gestores de empresas brasileiras listadas na Brasil Bolsa Balcão (B3). Foi estabelecido um ranking conforme os valores de P\&D e do ativo intangível divulgados pelas empresas. Este ranking se justifica pelo fato da pesquisa focalizar a capacidade de inovação das empresas em sentido duplo (inovação radical e incremental). Os projetos que traduzem a capacidade de inovação das empresas são identificados pelos itens de P\&D e Ativo Intangível, evidenciados nas demonstrações financeiras. A população da pesquisa compreende as 100 primeiras empresas do ranking.

Os contatos dos gestores destas empresas foram realizados pela rede LinkedIn, com envio de aproximadamente 5.000 convites para os cargos de Chief Executive Officer (CEO), Diretor Financeiro, Controller, Gerente, Supervisor e Coordenador, dos quais 1.136 aceitaram integrar a rede social e participar da pesquisa. Foi enviado o link da pesquisa, no período de fevereiro de 2019 até outubro de 2019, e obteve-se 128 respostas, mas 4 estavam incompletas, portanto, a amostra final compõe-se de 124 gestores de alto escalão e nível hierárquico médio.

Esta quantidade de respostas possibilita realizar os procedimentos estatísticos previstos, visto que satisfaz os critérios do tamanho do efeito (médio de 0,15); o nível de significância $(\alpha=5 \%)$; e o poder da amostra $(1-\beta=$ 0,8), atestados pelo $G^{*}$ power (Faul, Erdfelder, Buchner \& Lang, 2009). Outro pressuposto examinado é o NonReponse Bias, que aponta possíveis distorções na amostra. O teste T e a comparação entre os 12 primeiros e os 12 últimos respondentes não indicou diferenças significativas ao nível de $5 \%$, o que sugere que não existe o viés da não resposta (Af Wåhlberg \& Poom, 2015). Avaliou-se ainda o viés do método comum pelo teste de fator único de Harman, em que o primeiro fator apresentou variância total explicada inferior a 0,5, o que confirma a ausência do viés do método comum. 


\subsection{Mensuração das variáveis e procedimentos de análise}

Os três construtos da pesquisa foram mensurados a partir de assertivas já validadas nos estudos de Simons e Peterson (2000) e Bedford et al. (2019). A percepção dos gestores quanto ao debate das medidas para mensuração do desempenho no contexto de empresas que buscam a inovação ambidestra foi ancorada em assertivas na escala Likert de cinco pontos.

O debate do PMS foi mensurado a partir de quatro assertivas do estudo de Bedford et al. (2019), que desenvolveram este construto considerando o uso interativo do SCG, que permite aferir o debate das medidas de desempenho (Henri, 2006; Naranjo-Gil \& Hartmann, 2007; Widener, 2007). Este construto buscou capturar o uso pelos gestores das medidas de desempenho para suas discussões nas decisões de investimento e planos de ação relativos aos esforços voltados à inovação radical e incremental. Por exemplo, em uma das assertivas, os gestores foram solicitados a indicar em uma escala de 1 (nunca) a 5 (sempre), se as medidas de desempenho são frequentemente discutidas em reuniões da equipe de gestores seniores.

O conflito cognitivo presume-se que interage com o debate do PMS pelo fato de gerar tensão nas agendas dos gestores e, por sua vez, criam-se ideias inovadoras que são intensificadas pelo nível de conflito cognitivo alcançado pelo gestor. Este construto foi desenvolvido por Simons e Peterson (2000) e teve como base o estudo de Jehn (1995). Compõe-se de quatro assertivas que buscam aferir a percepção dos gestores sobre o efeito do PMS na geração de conflito cognitivo. Por exemplo, em uma das assertivas os gestores foram solicitados a indicar em uma escala de 1 (nenhuma) a 5 (muitíssimas) o quanto os membros da equipe de gestores possuem discordâncias sobre o conteúdo das decisões estratégicas da empresa. Esta variável é moderadora do modelo proposto. A variável moderadora pode alterar a força ou a direção da relação entre duas variáveis (Hair Jr, Hult, Ringle \& Sarstedt, 2016).

A inovação ambidestra é utilizada como um construto multidimensional, que engloba aspectos da inovação radical e incremental (Bedford et al., 2019). Assim, foi solicitado avaliar a capacidade dos gestores de efetivamente alcançarem objetivos concorrentes de inovação incremental (melhoria de produtos/serviços já existentes) e inovação radical (produtos novos, que requerem mudança de trajetória). O construto inovação ambidestra compõese de oito assertivas, sendo as quatro primeiras de inovação incremental e as quatro últimas de inovação radical. $\mathrm{Na}$ inovação incremental, o gestor foi solicitado a indicar em uma escala de 1 (discordo totalmente) a 5 (concordo totalmente) se a empresa frequentemente introduziu novos produtos/serviços incrementais nos últimos três anos. $\mathrm{Na}$ inovação radical, o gestor foi solicitado a indicar, se em comparação com os principais concorrentes, a sua empresa introduziu mais produtos/serviços radicalmente novos nos últimos três anos. Essas assertivas foram extraídas do estudo de Bedford et al. (2019), que as adaptaram de Atuahene-Gima (2005) e Lin, McDonough, Lin e Lin (2013).

Para testar as hipóteses utilizou-se a técnica de modelagem de equações estruturais (SEM) estimada a partir dos Mínimos Quadrados Parciais (PLS). A partir do software SmartPLS versão 3, determinaram-se: o algoritmo PLS, o bootstrapping e o blindfolding. A técnica algoritmo PLS, que avalia o modelo de mensuração, seguiu a definição de 300 iterações com 7 critérios de paragem. Para confirmar a confiabilidade os construtos devem apresentar índices de alfa de Cronbach e confiabilidade composta superiores a 0,70 (Hair Jr et al., 2016). Para confirmar a validade é necessário que a variância média extraída (Average Variance Extracted - AVE) seja superior a 0,50 (Hair Jr et al., 2016) e o critério Fornell e Larcker atendido. Para constatar as relações entre os construtos e a acurácia do modelo estrutural aplicaram-se as técnicas bootstrapping e blindfolding (Hair Jr et al., 2016). Como a variável moderadora é continua, seguiu-se a abordagem da moderação por termos de interação (Hair Jr et al., 2016).

\section{ANÁLISE DOS RESULTADOS}

\subsection{Modelo de mensuração}

A PLS-SEM foi realizada em duas etapas. Na primeira explorou-se o modelo de mensuração, que analisa os critérios de validade (convergente e discriminante) e confiabilidade (interna e externa), a fim de atestar as medidas dos construtos (Hair Jr et al., 2016). Na sequência, testou-se o modelo estrutural. Na Tabela 1 apresentamse os resultados do modelo de mensuração. 
Tabela 1. Modelo de mensuração

\begin{tabular}{cccccccc}
\hline Construtos & $\begin{array}{c}\text { Alfa de } \\
\text { Cronbach }\end{array}$ & $\begin{array}{c}\text { Confiabilidade } \\
\text { composta }\end{array}$ & AVE & $\mathbf{R}^{2}$ & \multicolumn{3}{c}{ Validade discriminante } \\
\cline { 5 - 8 } & 0,876 & 0,923 & 0,800 & - & 0,894 & & DPMS \\
\hline Conflito Cognitivo & 0,914 & 0,930 & 0,627 & 0,12 & 0,090 & 0,791 & \\
Inovação Ambidestra & 0,757 & 0,841 & 0,596 & & $-0,154$ & 0,318 & 0,772 \\
Debate do PMS & 0,75 &
\end{tabular}

Fonte: elaboração própria.

Nota: Alfa de Cronbach >0,70; Confiabilidade composta $>0,70$; Variância Média Extraída $(\mathrm{AVE})>0,50$. CG $=$ Conflito Cognitivo; IA = Inovação Ambidestra; DPMS = Debate do PMS.

Conforme a Tabela 1, os valores do alfa de Cronbach são maiores que 0,70, assim, atesta-se a confiabilidade do modelo e assegura-se que as respostas dos gestores não apresentam vieses (Hair Jr et al., 2016). Entre os construtos a inovação ambidestra apresentou o maior alfa de Cronbach $(0,914)$, seguido do conflito cognitivo $(0,876)$ e o debate do PMS (0,757). O item (CG_4) com valor inferior a 0,4 foi excluído do modelo. Hair Jr et al. (2016) apontam que a exclusão de um item pode ocorrer desde que leve ao aumento da AVE. Os critérios de validade convergente do modelo foram atendidos, visto que a AVE dos construtos está acima do limiar de 0,50 (Hair Jr et al., 2016). A validade discriminante pode ser realizada pela matriz das cargas cruzadas (cross loading) ou pelo critério de Fornell e Larcker, cujos critérios evidenciaram o quanto os construtos são distintos entre si.

\subsection{Modelo estrutural}

O modelo estrutural permite testar as hipóteses e verificar as relações entre os construtos conforme um diagrama de caminhos estatisticamente válidos (Hair Jr et al., 2016). Para tal executou-se a técnica bootstrapping, tendo como parâmetro 5.000 subamostras e interações, intervalo de confiança com enviesamento corrigido e acerelado (bias-corrected and accelerated) e teste ao nível de significância de 5\% (Hair Jr et al., 2016). Assim obteve-se o tamanho e significância dos coeficientes de caminho. Também determinou-se por meio da técnica blindfolding a validação preditiva do modelo indicado pelo coeficiente de determinação de Pearson $\left(\mathrm{R}^{2}\right)$ e observou-se a Relevância Preditiva pelo indicador de Stone-Geisser $\left(\mathrm{Q}^{2}\right)$. Na Tabela 2 apresentam-se as relações entre os construtos.

Tabela 2. Coeficiente de caminhos e avaliação do modelo estrutural

\begin{tabular}{lcccc}
\hline Relação entre os construtos & $\boldsymbol{\beta}$ & T-value & P-value & Hipóteses \\
\hline Debate do PMS $\rightarrow$ Inovação Ambidestra & 0,289 & 3,747 & $0,000 * * *$ & $\mathrm{H}_{1}$ Confirma \\
Conflito Cognitivo $\rightarrow$ Inovação Ambidestra & 0,175 & 1,637 & $0,050^{* *}$ & $\mathrm{H}_{2}$ Confirma \\
Debate do PMS X Conflito Cognitivo $\rightarrow$ Inovação Ambidestra & 0,325 & 5,085 & $0,000 * *$ & \\
\hline
\end{tabular}

Fonte: elaboração própria.

Nota: $\mathrm{N}=124$. Significância ao nível de $* \mathrm{p}<0,10 ; * * \mathrm{p}<=0,05 ; * * * \mathrm{p}<0,01$. R2 = Inovação Ambidestra $(0,12)$; Q2 $=$ Inovação Ambidestra $(0,12)$.

A hipótese $\mathrm{H}_{1}$ postulou que o debate do PMS exerce influência sobre a inovação ambidestra. Observase na Tabela 2 que o debate do PMS mostrou-se associado positiva e significativamente à inovação ambidestra $(0,289, \mathrm{p}<0,01)$, o que suporta a não rejeição da $\mathrm{H}_{1}$, ao nível de significância de $1 \%$. Depreende-se que o debate do PMS leva ao aumento da capacidade das organizações de inovar, o que indica que as medias de desempenho precisam ser alinhadas aos planos sobre os esforços relativos à capacidade de criação de novas ideias e que geram novos produtos ou serviços.

Na hipótese $\mathrm{H}_{2}$, presume-se que o conflito cognitivo modera a relação entre o debate do PMS e a inovação ambidestra (radical e incremental). Os resultados evidenciam que o conflito cognitivo modera a relação entre o debate do PMS e a inovação ambidestra $(0,325$, p $<0,01)$, o que suporta a não rejeição da $\mathrm{H}_{2}$ ao nível de significância de $1 \%$. Essas evidências denotam que a interação entre o debate do PMS e o conflito cognitivo levam a maior inovação ambidestra, ou seja, as discussões entre os gestores ocasionam conflito cognitivo, o que favorece a criação de novas ideias, que, por sua vez, direcionam esforços para o aumento da capacidade inovadora das empresas.

A avaliação da validade preditiva apresenta-se um coeficiente de determinação de Pearson ( $\left.\mathrm{R}^{2}\right)$ de $12 \%$, o que indica que as variáveis debate do PMS e conflito cognitivo trazem moderada explicação à inovação ambidestra. A relevância preditiva de $12 \%$, indicada pelo $\mathrm{Q}^{2}$, atesta acurácia do modelo, que quanto maior que zero melhor (Hair Jr et al., 2016). 


\subsection{Discussão dos resultados}

As discussões em torno dos principais achados da pesquisa visam analisar os efeitos do debate do PMS e do conflito cognitivo na inovação ambidestra. Entre as dimensões constitutivas do uso interativo do SCG consta o debate do PMS, que permite o estabelecimento de padrões de comunicação entre o top management team (TMT), que leva a maior interação entre os mesmos. Assim, a confirmação da hipótese $\mathrm{H}_{1}$ sugere que nas empresas pesquisadas o debate do PMS facilita o processo de comunicação entre os gestores e fornece liberdade equilibrada, que favorecem o processo de inovação (Davila et al., 2009; Bedford et al., 2019). Esses achados corroboram que o SCG estimula a capacidade criativa do indivíduo e a aprendizagem organizacional, os quais promovem a inovação nas organizações (Simons, 1995; Davila, 2000; Lopez-Valeiras et al., 2016). Infere-se que é pelo debate frequente das medidas de desempenho que as organizações se antecipam em relação aos seus concorrentes e introduzem mais produtos e serviços radicalmente novos no mercado.

Diferentes opiniões entre os gestores contribuem para que as decisões sejam tomadas com maior qualidade (Dreu \& West, 2001). Assim, é importante que o debate seja constante, já que pode estreitar o relacionamento entre os gestores e aumentar a comunicação (Simons, 1995). Também é imprescindível que o uso do PMS facilite o processo de tomada de decisão. Nas empresas pesquisadas observa-se que é recorrente o debate em relação ao PMS e isso tem levado ao aumento de esforços à inovação incremental e radical e impactado no crescimento das vendas de produtos e serviços novos. As empresas estão preocupadas não apenas em gerar mudanças nos seus produtos e serviços para atenderem às demandas já conhecidas de seus clientes (Garcia \& Calantone, 2002), mas também em criar mudanças tecnológicas que geram demandas não conhecidas e nem atendidas pelo mercado (Jansen et al., 2006).

$\mathrm{Na}$ hipótese $\mathrm{H}_{2}$, que previa moderação do conflito cognitivo na relação entre o debate do PMS e a inovação ambidestra, primeiramente confirmou-se relação direta e significativa ao nível de $5 \%(0,175, p=0,050)$ entre o conflito cognitivo e a inovação ambidestra. Essas evidências se coadunam com as de Dreu (2006), de que a presença de conflito cognitivo incita os indivíduos a examinarem os problemas organizacionais, propicia maior envolvimento destes em atividades criativas e aumenta a inovação. Na sequência, confirmou-se o papel moderador do conflito cognitivo na relação entre o debate do PMS e a inovação ambidestra, ao nível de significância de $1 \%$ $(0,325, \mathrm{p}<0,01)$. Na Figura 2 ilustra-se o efeito da interação entre o debate do PMS e o conflito cognitivo.

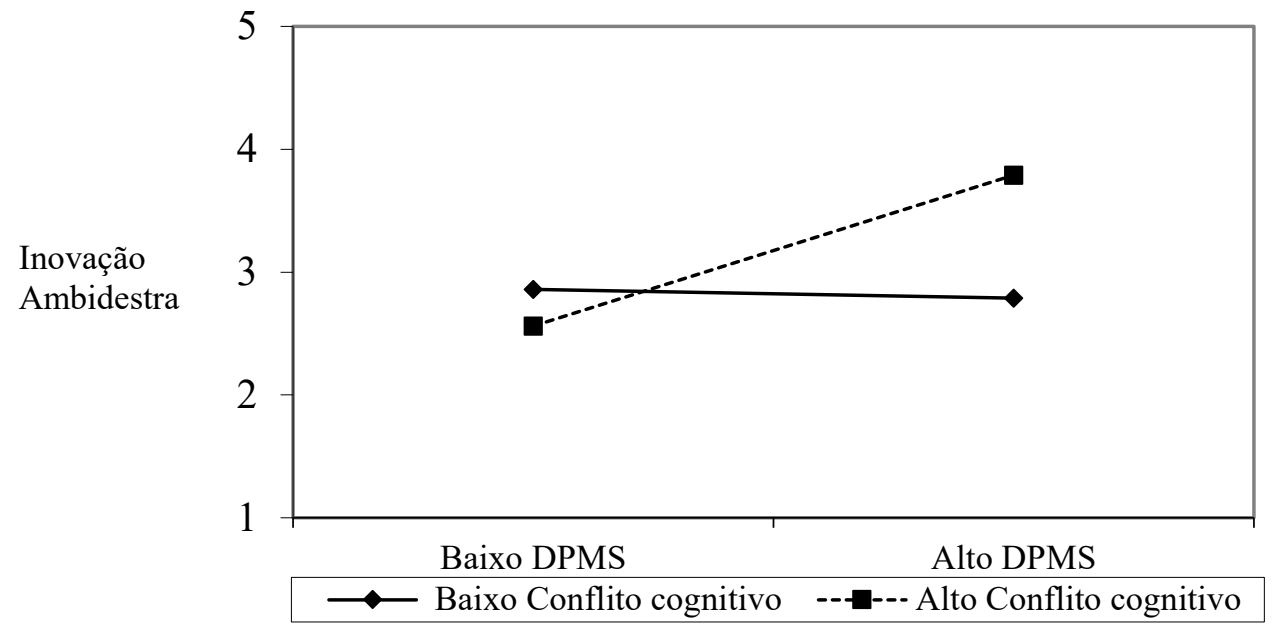

Figura 2. Efeito da interação entre o debate do PMS (DPMS) e o conflito cognitivo

Verifica-se na Figura 2 que à medida em que aumenta o conflito cognitivo, mais forte é o efeito do debate do PMS na inovação ambidestra, ou seja, a existência de diferentes opiniões entre os gestores durante o processo decisório intensifica a relação entre o debate do PMS e a inovação ambidestra. Para que o debate do PMS possa facilitar a troca de informações entre os gestores (Bedford et al., 2019), estreitar o relacionamento entre o gestores (Simons, 1995), gerar maior comunicação e decisões mais assertivas em direção ao alcance da inovação incremental e radical (Bedford et al., 2019) é importante que os gestores desenvolvam a capacidade de resolução de problemas, aprendizagem e de criação de novas ideias, diante das divergências de opinião (Dreu, 2006). Isso permite que esses esforços conjuntos se alinhem e levem ao aumento da capacidade da organização gerar mudanças tecnológicas nos seus produtos e serviços (Garcia \& Calantone, 2002; Jansen et al., 2006). 
Infere-se que a presença do conflito cognitivo decorrente das diferenças de opinião entre os gestores é importante para o alcance dos objetivos organizacionais. No entanto, é preciso que os gestores durante o debate do PMS não incorram em conflito afetivo, visto que este é decorrente de discordância que leva à insatisfação pessoal e não é considerado benéfico para o ambiente de trabalho, os gestores e a organização como um todo (Dreu, 2006; Desivilya et al., 2010). Os resultados desta pesquisa apoiam o entendimento de que uma melhor compreensão sobre as discussões das medidas de desempenho e a existência de discordâncias orientadas para as tarefas e estratégias relativas aos projetos de investimento em P\&D melhoram o processo de tomada de decisão e o desempenho organizacional.

\section{CONCLUSÕES}

Os resultados da pesquisa revelaram que o debate do PMS exerce influência positiva e significativa na inovação ambidestra, o que indica que as discussões das medidas de desempenho propiciam o envolvimento dos gestores na busca de soluções orientadas para maior visibilidade de esforços voltados à mudanças tecnológicas nos produtos e serviços. Evidenciaram também que o conflito cognitivo modera a relação entre o debate do PMS e a inovação ambidestra, o que sugere que é importante que o debate do PMS ocorra em um ambiente com diferenças de opiniões entre os gestores, pelo fato de gerar ideias criativas, aprendizagem e aumentar a inovação incremental e radical.

A pesquisa apresenta implicações teóricas ao tratar da relação entre o PMS e a inovação a partir de uma construção multidimensional da inovação (incremental e radical), portanto se difere de estudos pregressos que investigaram a relação entre SCG e a inovação a partir de uma construção unidimensional. Assim, contribui-se para avanços na literatura sobre estudos em contextos organizacionais que visam à inovação ambidestra relacionada ao SCG. Contribui também para a literatura sobre conflito, ao reforçar que a presença de conflito cognitivo no ambiente de trabalho promove resultados benéficos para a organização, pelo fato de intensificar a relação entre o SCG e a inovação, desde que a dimensão do debate das medidas de desempenho seja focada no uso interativo do PMS. Estudos prévios consideraram o conflito cognitivo como variável mediadora, enquanto neste estudo encontraram-se evidências de que esta variável também funciona como moderadora.

O estudo também apresenta implicações práticas para as organizações ao revelar impacto positivo e significativo do debate do PMS na inovação ambidestra. Os resultados podem instigar as empresas a fomentar um ambiente de debate entre os gestores, principalmente nas agendas de elaboração dos planos e ações da organização. Diferentes opiniões entre os gestores favorecem aproximação entre eles, aumenta a aprendizagem e a capacidade de criação de novas ideias por parte dos gestores com vistas na resolução de problemas. Dessa maneira, as agendas dos gestores tendem a protagonizar estratégias que dão visibilidade às melhorias tecnológicas em produtos e serviços. Esse esforço precisa ser direcionado de maneira dupla (radical e incremental). As empresas precisam se antecipar para liderar a concorrência, buscando melhorias relacionadas ao design, função, preço, quantidades e características dos seus produtos e serviços, tanto de forma radical quanto incremental.

No entanto, os resultados da pesquisa devem ser interpretados com parcimônia devido as limitações impostas no seu delineamento. Relações significativas foram observadas entre os construtos propostos a partir da análise de caminhos. Todavia, recomenda-se a adoção de métodos experimentais a fim de verificar relações causais entre os construtos propostos. O conflito cognitivo foi considerado como variável moderadora, por conseguinte, pesquisas futuras podem identificar se o conflito afetivo exerce influência negativa na criatividade ou na capacidade da organização em gerar mudanças tecnológicas nos seus produtos e serviços. Recomenda-se ainda que o conflito cognitivo seja considerado uma variável de efeito quadrático, já que pode apresentar efeito duplo, até certo ponto positivo e depois negativo. 


\section{REFERÊNCIAS}

Af Wåhlberg, A.E., \& Poom, L. (2015). An empirical test of nonresponse bias in internet surveys. Basic and Applied Social Psychology, 37(6), 336-347. DOI: https://doi.org/10.1080/01973533.2015.1111212

Amason, A.C., \& Mooney, A.C. (1999). The effects of past performance on top management team conflict in strategic decision making. International Journal of Conflict Management, 10(4), 340-359.

Anthony, R.N. (1965). Planning and control systems: A framework for analysis. Massachusetts: Harvard University.

Atuahene-Gima, K. (2005). Resolving the capability-rigidity paradox in new product innovation. Journal of Marketing, 69(4), 61-83. DOI: https://doi.org/10.1509/jmkg.2005.69.4.61

Bedford, D.S. (2015). Management control systems across different modes of innovation: implications for firm performance. Management Accounting Research, 28(3), 12-30. DOI: https://doi.org/10.1016/j. mar.2015.04.003

Bedford, D.S., Bisbe, J., \& Sweeney, B. (2019). Performance measurement systems as generators of cognitive conflict in ambidextrous firms. Accounting, Organizations and Society, 72(1), 21-37. DOI: https://doi. org/10.1016/j.aos.2018.05.010

Bedford, D.S., \& Speklé, R.F. (2018). Construct validity in survey-based management accounting and control research. Journal of Management Accounting Research, 30(2), 23-58. DOI: https://doi.org/10.2308/jmar51995

Bisbe, J., \& Malagueño, R. (2009). The choice of interactive control systems under different innovation management modes. European Accounting Review, 18(2), 371-405.

Bisbe, J., \& Malagueño, R. (2015). How control systems influence product innovation processes: Examining the role of entrepreneurial orientation. Accounting and Business Research, 45(3), 356-386. DOI: https://doi. org/10.1080/09638180902863803

Bisbe, J., \& Otley, D. (2004). The effects of the interactive use of management control systems on product innovation. Accounting, Organizations and Society, 29(8), 709-737. DOI: https://doi.org/10.1016/j.aos.2003.10.010

Calton, J.M., \& Payne, S.L. (2003). Coping with paradox: Multistakeholder learning dialogue as a pluralist sensemaking process for addressing messy problems. Business \& Society, 42(1), 7-42. DOI: https://doi. org/10.1177/0007650302250505

Chenhall, R.H. (2003). Management control systems design within its organizational context: findings from contingency-based research and directions for the future. Accounting, Organizations and Society, 28(2-3), 127-168. DOI: https://doi.org/10.1016/S0361-3682(01)00027-7

Chenhall, R.H., Kallunki, J.P., \& Silvola, H. (2011). Exploring the relationships between strategy, innovation, and management control systems: the roles of social networking, organic innovative culture, and formal controls. Journal of Management Accounting Research, 23(1), 99-128. DOI: https://doi.org/10.2308/jmar-10069

Davila, A., Foster, G., \& Oyon, D. (2009). Accounting and control, entrepreneurship and innovation: Venturing into new research opportunities. European Accounting Review, 18(2), 281-311. DOI: https://doi. org/10.1080/09638180902731455

Davila, T. (2000). An empirical study on the drivers of management control systems' design in new product development. Accounting, Organizations and Society, 25(4-5), 383-409. DOI: https://doi.org/10.1016/S03613682(99)00034-3

Davila, T., Epstein, M., \& Shelton, R. (2012). Making innovation work: How to manage it, measure it, and profit from it. Upper Saddle River: FT press.

Dekker, H.C., Groot, T., \& Schoute, M. (2012). A balancing act? The implications of mixed strategies for performance measurement system design. Journal of Management Accounting Research, 25(1), 71-98. DOI: https://doi.org/10.2308/jmar-50356

Desivilya, H.S., Somech, A., \& Lidgoster, H. (2010). Innovation and conflict management in work teams: The effects of team identification and task and relationship conflict. Negotiation and Conflict Management Research, 3(1), 28-48. DOI: https://doi.org/10.1111/j.1750-4716.2009.00048.x

Dreu, C.K. (2006). When too little or too much hurts: Evidence for a curvilinear relationship between task conflict and innovation in teams. Journal of Management, 32(1), 83-107. DOI: https://doi.org/10.1177/0149206305277795 
Dreu, C.K., \& West, M.A. (2001). Minority dissent and team innovation: The importance of participation in decision making. Journal of Applied Psychology, 86(6), 1191-1201. DOI: 10.1037/0021-9010.86.6.1191

Faul, F., Erdfelder, E., Buchner, A., \& Lang, A.G. (2009). Statistical power analyses using G* Power 3.1: Tests for correlation and regression analyses. Behavior Research Methods, 41(4), 1149-1160.

Ferreira, A., \& Otley, D. (2009). The design and use of performance management systems: An extended framework for analysis. Management Accounting Research, 20(4), 263-282. DOI: https://doi.org/10.1016/j. mar.2009.07.003

Flamholtz, E.G., Das, T.K., \& Tsui, A.S. (1985). Toward an integrative framework of organizational control. Accounting, Organizations and Society, 10(1), 35-50. DOI: https://doi.org/10.1016/0361-3682(85)90030-3

Garcia, R., \& Calantone, R. (2002). A critical look at technological innovation typology and innovativeness terminology: a literature review. Journal of Product Innovation Management: An International Publication of the Product Development \& Management Association, 19(2), 110-132. DOI: https://doi.org/10.1111/15405885.1920110

Hair Jr, J.F., Hult, G.T.M., Ringle, C., \& Sarstedt, M. (2016). A primer on partial least squares structural equation modeling (PLS-SEM). California: Sage publications.

Henri, J.F. (2006). Management control systems and strategy: a resource-based perspective. Accounting, Organizations and Society, 31(6), 529-558. DOI: https://doi.org/10.1016/j.aos.2005.07.001

Jansen, J.J., Van Den Bosch, F.A., \& Volberda, H.W. (2006). Exploratory innovation, exploitative innovation, and performance: Effects of organizational antecedents and environmental moderators. Management Science, 52(11), 1661-1674. DOI: https://doi.org/10.1287/mnsc.1060.0576

Jehn, K.A. (1995). A multimethod examination of the benefits and detriments of intragroup conflict. Administrative Science Quarterly, 40 (2) 256-282.

Jehn, K.A. (1997). A qualitative analysis of conflict types and dimensions in organizational groups. Administrative Science Quarterly, 42 (3)530-557.

Kim, D.Y., Kumar, V., \& Kumar, U. (2012). Relationship between quality management practices and innovation. Journal of Operations Management, 30(4), 295-315. DOI: https://doi.org/10.1016/j.jom.2012.02.003

Koberg, C.S., Detienne, D.R., \& Heppard, K.A. (2003). An empirical test of environmental, organizational, and process factors affecting incremental and radical innovation. The Journal of High Technology Management Research, 14(1), 21-45. DOI: https://doi.org/10.1016/S1047-8310(03)00003-8

Lamberti, L., \& Noci, G. (2010). Marketing strategy and marketing performance measurement system: Exploring the relationship. European Management Journal, 28(2), 139-152. DOI: https://doi.org/10.1016/j. emj.2009.04.007

Lin, H.E., McDonough III, E.F., Lin, S.J., \& Lin, C.Y.Y. (2013). Managing the exploitation/exploration paradox: The role of a learning capability and innovation ambidexterity. Journal of Product Innovation Management, 30(2), 262-278. DOI: https://doi.org/10.1111/j.1540-5885.2012.00998.x

Lopez-Valeiras, E., Gonzalez-Sanchez, M.B., \& Gomez-Conde, J. (2016). The effects of the interactive use of management control systems on process and organizational innovation. Review of Managerial Science, 10(3), 487-510. DOI: $10.1007 / \mathrm{s} 11846-015-0165-9$

Malmi, T., \& Brown, D.A. (2008). Management control systems as a package-Opportunities, challenges and research directions. Management Accounting Research, 19(4), 287-300. DOI: https://doi.org/10.1016/j. mar.2008.09.003

Naranjo-Gil, D., \& Hartmann, F. (2007). Management accounting systems, top management team heterogeneity and strategic change. Accounting, Organizations and Society, 32(7-8), 735-756. DOI: https://doi.org/10.1016/j. aos.2006.08.003

Sarkees, M., \& Hulland, J. (2009). Innovation and efficiency: It is possible to have it all. Business horizons, 52(1), 45-55. DOI: https://doi.org/10.1016/j.bushor.2008.08.002

Simons, R. (1995). Levers of control: How managers use innovative control systems to drive strategic renewal. Massachusetts: Harvard Business Press. 
Simons, T.L., \& Peterson, R.S. (2000). Task conflict and relationship conflict in top management teams: the pivotal role of intragroup trust. Journal of Applied Psychology, 85(1), 102-111. DOI: 10.1037/0021-9010.85.1.102

Tjosvold, D., Poon, M., \& Yu, Z.Y. (2005). Team effectiveness in China: Cooperative conflict for relationship building. Human Relations, 58(3), 341-367. DOI: https://doi.org/10.1177/0018726705053426

West, M.A. (2002). Sparkling fountains or stagnant ponds: An integrative model of creativity and innovation implementation in work groups. Applied Psychology, 51(3), 355-387. https://doi.org/10.1111/14640597.00951

\section{Como citar este artigo}

Monteiro, J. J.; \& Beuren, I. M. (2020). Efeitos do debate do sistema de mensuração de desempenho e do conflito cognitivo na inovação ambidestra. Revista de Contabilidade e Organizações, 14:e170418. DOI: http://dx.doi.org/10.11606/issn.1982-6486.rco.2020.170418 